\title{
ReMed: Ärzte für Ärzte
}

\author{
Ärztinnen und Ärzte sind beruflich stark gefordert - manchmal so stark, dass \\ es zu gesundheitlichen und psychischen Beschwerden kommt. Wenn noch \\ Schwierigkeiten im privaten Umfeld dazukommen, scheint die Situation plötz- \\ lich ausweglos. In solchen Krisen bietet ReMed, das Unterstützungsnetzwerk, \\ rasch und kompetent Unterstützung.
}

Jürg Beutler

Kommunikation FMH

ReMed berät und begleitet Ärztinnen und Ärzte in kritischen Momenten. Das Unterstützungsnetzwerk meldet sich bei jeder Kontaktaufnahme innerhalb von 72 Stunden und bespricht unverbindlich und vertraulich die persönliche Situation und individuelle Handlungsmöglichkeiten. ReMed ist an das Arztgeheimnis gebunden.

Benötigen Sie Unterstützung? Oder eine Ärztin, ein Arzt aus Ihrem Umfeld?

Dann kontaktieren Sie ReMed: 24-Stunden-Hotline 08000 73633, info@swiss-remed.ch, www.swiss-remed.ch tielle Ängste.

\section{Erfahrungsbericht}

Ein 55jähriger Hausarzt, der eine Doppelpraxis führt und als Heimarzt in einem Pflegeheim tätig ist, berichtet von seinen persönlichen Erfahrungen: Nebst der grossen beruflichen Belastung plagen ihn als Vater

«Meine zunehmende Erschöpfung hatte sich schon lange abgezeichnet: Ich legte Bücher ungelesen weg, ich wurde vergesslicher, machte Fehler und trank auch mehr Alkohol. Vordergründig funktionierte ich gut. Doch meine Praxisassistentin machte mich immer öfter auf Aussetzer aufmerksam. Das erschreckte mich. Und dass mich die Geschichten meiner Patienten plötzlich nicht mehr interessierten, traf mich in meiner ärztlichen Identität. Als Familienvater plagten mich zudem unbegründete existentielle Ängste. Ich begann an den Börsen zu spekulieren, das nächtelange Beobachten der Kurse wurde zum Zwang.

Der Weg aus der Krise war nicht einfach. Ich realisierte, dass eine Auszeit notwendig war und dass ich professionelle Hilfe brauchte. Ich fand sie bei meinem Hausarzt. Mein Praxiskollege übernahm vorübergehend meine Patienten, so dass ich wieder Zeit für mich hatte. Allmählich reifte in mir der Entschluss, meine Hausarztpraxis nach 20 Jahren aufzugeben, um so mein Arbeitspensum radikal zu reduzieren. Meine Tätigkeit an der Klinik führte ich fort, denn dort fühlte ich mich wohl: Die Arbeitszeiten sind klar geregelt, die Zusammenarbeit mit jungen Leuten ist bereichernd. Heute geniesse ich die neu ge- wonnene Zeit, und es geht mir gut. Entscheidend dafür war, dass ich mir meine Krise eingestanden und auch in meinem Qualitätszirkel darüber gesprochen habe.»

\section{Interview mit dem Projektleiter}

Michael Peltenburg, Sie sind Projektleiter von ReMedhätte sich der Hausarzt, von dem der Erfahrungsbericht handelt, an das Unterstützungsnetzwerk wenden können?

Ja. ReMed ist in den Kantonen Neuenburg und Thurgau als Pilotprojekt etabliert. Die Kolleginnen und Kollegen in diesen Kantonen sind über das Angebot informiert. Selbstverständlich nimmt ReMed aber auch Anfragen aus der übrigen Schweiz via Hotline oder Homepage entgegen.

Was hätte ReMed in diesem Fall unternommen? ReMed hätte in diesem recht typischen Fall professionelle Hilfe und eine konkrete Entlastung in der Praxis vermittelt. Ich denke, dass ReMed nach einer gemeinsamen Betrachtung der Situation mit diesem Kollegen eine spezifische Behandlung zur Bewältigung seines Burn-outs angestrebt hätte. Zudem wären wohl auch die Möglichkeit einer Beratung zur Verbesserung des Managements sowie eine vorübergehende Entlastung in der Praxis diskutiert worden.

Ganz allgemein: Wer soll sich an ReMed wenden? Ärztinnen und Ärzte, die sich in einer Krise befinden. Der Kontakt kann ebenfalls über ihre Angehörigen und Mitarbeitenden erfolgen. ReMed spricht natürlich auch jene Ärztinnen und Ärzte an, die sich begleiten lassen wollen, bevor es zu einer Krise kommt, um sich so die Freude am Beruf möglichst lange zu erhalten. Das Stichwort heisst hier Mentoring. Zusätzliche Informationen dazu sind auf www.swiss-remed.ch zu finden.
Korrespondenz:

Dr. med. Michael Peltenburg

Facharzt für Allgemeinmedizin FMH

michael.peltenburg@hin.ch 